\title{
Monitoring oral anticoagulant treatment with the TAS near-patient test system: comparison with conventional thromboplastins
}

\author{
S Kitchen, F E Preston
}

\begin{abstract}
Background-A number of instruments have been developed for determination of prothrombin time (PT) and International Normalised Ratio (INR) at locations not limited to central laboratories.

Aim-To evaluate one such portable instrument, the Thrombolytic Assessment System (TAS), which can be used in a near-patient setting.
\end{abstract}

Methods-Samples from 20 normal subjects and 48 patients treated with warfarin for venous thromboembolic disease were studied. The warfarin group was divided into: initiation phase $(n=10)$, combined warfarin and heparin $(n=10)$, stabilised therapy $(n=20)$, and over anticoagulated patients $(n=8)$. PTs and INRs were determined in each group using three conventional thromboplastins (Diagen Activated, Manchester Reagent, and Instrumentation Laboratory) and two TAS techniques (whole blood or plasma). An independent International Sensitivity Index (ISI) calibration of the TAS system was performed. Results-Calculated ISIs for the TAS were 1.028 and 0.984 for plasma and whole blood analysis, respectively, compared with manufacturer's values of 0.98 and 0.97. INR results with TAS (whole blood) were $11 \%$ less than those obtained with Diagen Activated $(p<0.01)$ and $16 \%$ less than those obtained with Instrumentation Laboratory ( $p<0.001$ ) when manufacturers' mean normal PT and ISI were used for TAS INRs. TAS (whole blood) results were similar to TAS plasma or Manchester Reagent results. The use of a locally determined mean normal prothrombin time (MNPT) improved agreement between TAS and the other reagents, abolishing the significant difference between INRs determined with TAS (whole blood) and Diagen Activated techniques.

Conclusion-The TAS system can be used with whole blood or plasma and produces similar INRs to those obtained with Diagen Activated or Manchester Reagent using manufacturer's ISI and a locally determined MNPT. Results were lower with TAS or Manchester Reagent compared with those obtained with Instrumentation Laboratory thromboplastin. (f Clin Pathol 1997;50:951-956)

Mr Kitchen.

Accepted for publication 2 September 1997
Keywords: INR; near patient testing; Thrombolytic Assessment System; prothrombin time
Oral anticoagulants are widely used for the prevention and treatment of thromboembolic disease. ${ }^{1}$ Optimal oral anticoagulant dosage regimens reflect a balance between the antithrombotic effects of these drugs and unwanted haemorrhagic side effects. Warfarin dose adjustments necessitate reliable laboratory monitoring. The test most commonly used for this purpose is the prothrombin time (PT) expressed as International Normalised Ratio (INR). The INR system is a standardised system of reporting that takes account of the variability in the responsiveness of thromboplastins to the defect induced by coumarin drugs. ${ }^{23}$ The theoretical advantage of this system is that the same INR is obtained irrespective of the laboratory reagent used, and its introduction has undoubtedly contributed to improvement in the laboratory control of oral anticoagulant treatment. ${ }^{4}$

The problems of large scale laboratory monitoring of oral anticoagulants particularly in an outpatient setting, have led to the recent development of a number of near-patient testing instruments. ${ }^{5-10}$

One such portable instrument is the Thrombolytic Assessment System (TAS) that can be used to determine PT and INR in a nearpatient setting. ${ }^{7}$ We compared INR results obtained with this instrument to those obtained using three thromboplastins in common use in the UK.

\section{Materials and methods}

SAMPLES

Venous blood samples were collected in the proportion 9 parts blood to 1 part $0.105 \mathrm{M}$ buffered trisodium citrate (Vacutainer, BectonDickinson Ltd, Cowley, Oxford, UK). One millilitre of whole blood was transferred to a stoppered plastic container and the remaining $4 \mathrm{ml}$ centrifuged at $2000 \times g$ for 10 minutes at room temperature. The citrated whole blood sample was tested using the TAS system only, whereas the citrated platelet poor plasma was tested using the TAS system and three conventional thromboplastins.

\section{PATIENT GROUPS}

Samples were collected from 48 patients receiving warfarin for venous thromboembolic disease, which comprised the following:

Initiation phase-10 patients during the first 10 days of treatment not receiving concurrent heparin at the time of sample collection. 
Table 1 Precision of PT and INR tests for the TAS system and conventional thromboplastins

\begin{tabular}{|c|c|c|c|c|c|c|}
\hline & & \multirow{2}{*}{$\begin{array}{l}\text { TAS system } \\
\text { whole blood }\end{array}$} & \multirow{2}{*}{$\begin{array}{l}\text { TAS system } \\
\text { plasma }\end{array}$} & \multicolumn{3}{|c|}{$\begin{array}{l}\text { Conventional thromboplastins } \\
\text { (plasma) }\end{array}$} \\
\hline & & & & $M R$ & $I L$ & $D A$ \\
\hline \multicolumn{7}{|c|}{ Within-assay precision } \\
\hline \multirow[t]{2}{*}{ Normal } & Mean PT (s) & 10.9 & 10.5 & 16.4 & 13.4 & 14.6 \\
\hline & $\mathrm{CV}(\%)$ & 3.6 & 1.0 & 3.6 & 1.0 & 2.3 \\
\hline \multirow{2}{*}{ Abnormal } & Mean INR & 2.75 & 2.93 & 2.62 & 3.07 & 2.59 \\
\hline & $\mathrm{CV}(\%)$ & 9.7 & 7.3 & 4.4 & 0.9 & 6.7 \\
\hline \multicolumn{7}{|c|}{ Between-assay precision } \\
\hline \multirow[t]{2}{*}{ Normal } & Mean PT (s) & - & 10.9 & 15.6 & 13.2 & 14.5 \\
\hline & $\mathrm{CV}(\%)$ & - & 2.4 & 3.3 & 1.9 & 3.7 \\
\hline \multirow[t]{2}{*}{ Abnormal } & Mean INR & - & 2.47 & 2.33 & 3.04 & 2.39 \\
\hline & $\mathrm{CV}(\%)$ & - & 7.9 & 9.9 & 4.2 & 7.7 \\
\hline
\end{tabular}

Means are average of 10 replicates. Samples used for within-assay precision were different for each technique; samples used for between-assay precision were the same for all techniques.

MR, Manchester Reagent; IL, Instrumentation Laboratory; DA, Diagen Activated.

Table 2 Prothrombin times of 20 normal subjects

\begin{tabular}{|c|c|c|c|c|c|}
\hline & \multirow{2}{*}{$\begin{array}{l}\text { TAS system } \\
\text { whole blood }\end{array}$} & \multirow{2}{*}{$\begin{array}{l}\text { TAS system } \\
\text { plasma }\end{array}$} & \multicolumn{3}{|c|}{ Conventional thromboplastins } \\
\hline & & & $M R$ & $I L$ & $D A$ \\
\hline Mean PT (s) & 10.6 & 10.7 & 15.5 & 13.0 & 14.3 \\
\hline Standard deviation & 1.12 & 0.93 & 0.94 & 0.82 & 0.86 \\
\hline
\end{tabular}

MR, Manchester Reagent; IL, Instrumentation Laboratory; DA, Diagen Activated.

Combined warfarin and heparin-10 patients receiving both warfarin and concurrent intravenous unfractionated sodium heparin treatment.

Stable warfarin therapy-20 patients stabilised on warfarin with no major change in INR for at least four weeks.

Over anticoagulated patients -8 patients with INRs $>4.5$ (as measured using Instrumentation Laboratory thromboplastin).

TAS INSTRUMENT: PRINCIPLE OF ANALYSIS

The TAS system consists of a microprocessor controlled analyser (Cardiovascular Diagnostic Inc, Research Triangle Park, North Carolina, USA) and individual disposable test cards. Clotting is measured in the presence of inert paramagnetic iron oxide particles in combination with a human placental thromboplastin containing calcium, dried and contained in a reaction chamber within the test card as described elsewhere. ${ }^{11}$

DETERMINATION OF INR: CONVENTIONAL THROMBOPLASTINS

All tests were performed between one and two hours after sample collection and the order of testing with different techniques (three conventional thromboplastins, TAS system with plasma, TAS system with whole blood) was

Table 3 Mean INRs determined using the TAS system and three conventional thromboplastins

\begin{tabular}{llllllll}
\hline Patient group & $n$ & $\begin{array}{l}\text { TAS whole } \\
\text { blood (1) }\end{array}$ & $\begin{array}{l}\text { TAS } \\
\text { plasma }\end{array}$ & MR & IL & DA & $\begin{array}{l}\text { TAS whole } \\
\text { blood (2) }\end{array}$ \\
\hline $\begin{array}{l}\text { Initiation of warfarin } \\
\text { Combined heparin and }\end{array}$ & 10 & 1.50 & 1.61 & 1.60 & 1.73 & 1.61 & 1.60 \\
$\quad$ warfarin & 10 & 1.85 & 1.93 & 2.23 & 2.05 & 2.27 & 2.01 \\
$\begin{array}{l}\text { Stable warfarin therapy } \\
\text { Over anticoagulated }\end{array}$ & 20 & 2.36 & 2.52 & 2.54 & 2.87 & 2.69 & 2.53 \\
$\quad$ patients & 8 & 7.08 & 8.14 & 6.76 & 7.05 & 7.93 & 7.59 \\
All groups combined* & 45 & 2.40 & 2.59 & 2.51 & 2.78 & 2.68 & 2.58 \\
\hline
\end{tabular}

TAS whole blood (1) was calculated using the manufacturer's MNPT (encoded into test cards); TAS whole blood (2) was calculated using the local MNPT

$\star$ Excluded three patients in whom INRs of $>10.0$ were recorded using at least one technique. MR, Manchester Reagent; IL, Instrumentation Laboratory; DA, Diagen Activated. varied. Prothrombin times were determined on plasma samples with the following conventional thromboplastins and techniques:

Manchester Reagent (MR), Manchester Thrombosis Research Foundation, Stockport, UK; batch 94/10, International Sensitivity Index (ISI) 1.12; manual technique

Diagen Activated (DA), Diagnostic Reagents, Thame, Oxfordshire, UK; batch RB 375, ISI 1.4; manual technique

Instrumentation Laboratory PT-Fib HS Plus (IL), Instrumentation Laboratory, Warrington, UK; batch I0940356, ISI 1.19; Automated Coagulation Laboratory (ACL) technique.

In accordance with common practice in UK laboratories reagents with a manual technique (MR and DA) underwent duplicate testing, whereas IL reagent underwent single tests. For each patient sample INRs were determined using the formula:

$$
\mathrm{INR}=\left(\frac{\mathrm{PT}}{\mathrm{MNPT}}\right)^{\mathrm{ISI}}
$$

where the mean normal prothrombin time (MNPT) was calculated as the geometric mean PT of 20 fresh normal plasmas for each technique. The ISIs used to determine INRs were as stated by the manufacturers.

DETERMINATION OF INR: TAS INSTRUMENT

Single test PT and INRs were determined using both plasma and whole blood samples for each patient. The analyser and test cards were used according to manufacturer's instructions. A measured volume of $35 \mu \mathrm{l}$ (whole blood or plasma) was used except where stated. The applied blood sample dissolves the dried human placental thromboplastin/calcium contained within the card. For the card batch used the following values had been encoded by the manufacturer: plasma, MNPT 10.9 seconds and ISI 0.98; whole blood, MNPT 11.4 seconds and ISI 0.97 .

INR values for the TAS system were those calculated and automatically displayed by the TAS instrument (that is, using the manufacturer's MNPT and ISI) unless otherwise stated. For INRs $>5$ as determined by the TAS system, only PT and PT ratios were displayed. In these cases the INR was calculated using the appropriate ISI and formula INR $=$ PT ratio ${ }^{\text {ISI }}$.

The importance of volume of applied blood drop on INR as determined using the TAS system was assessed. Whole blood samples from 10 patients on warfarin were analysed twice, first using a measured $35 \mu \mathrm{l}$ volume of whole blood, and second using an unmeasured droplet of unknown volume applied using a disposable plastic Pasteur pipette. The order of testing with the two methods was varied.

\section{Use of a local mean normal prothrombin time}

Two INRs were calculated for each patient's whole blood PT determined using the TAS instrument. The first was derived from the manufacturer's values for ISI and MNPT, encoded in the test cards. The second was calculated using a locally determined MNPT (geometric mean PT of 20 fresh normal samples). 


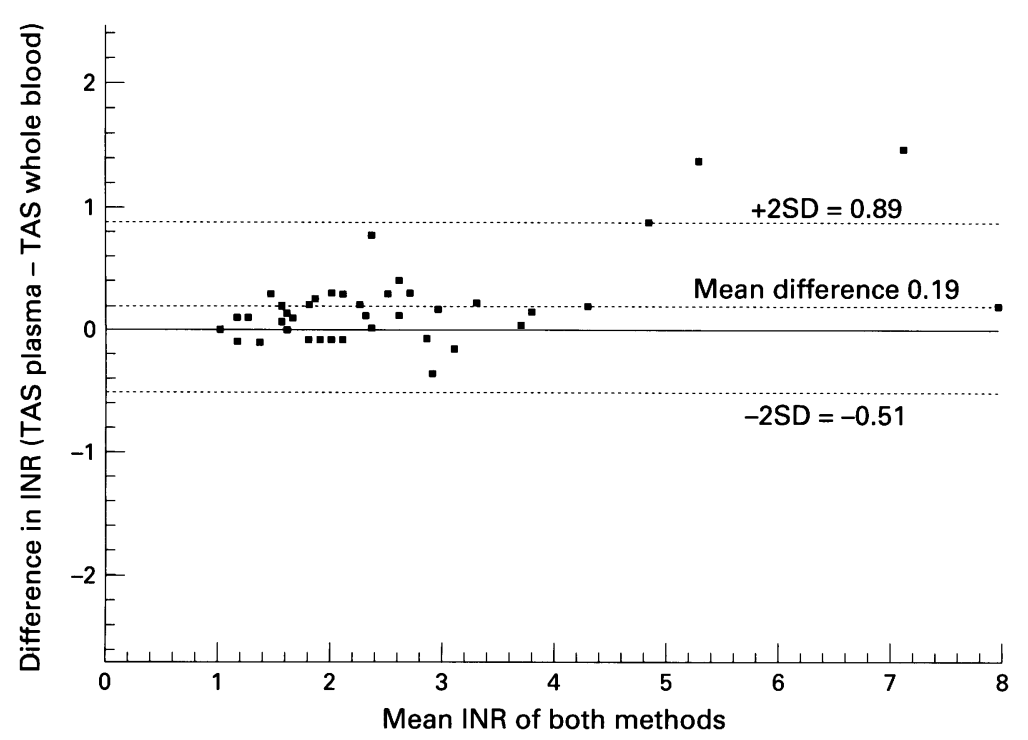

Figure 1 Difference in INRs of matched plasma and whole blood samples determined with the TAS instrument. The dotted lines are the mean (2 SD) of the differences in INR between the two methods. The solid line represents zero difference.

International sensitivity index of TAS PT cards The ISI of this batch of TAS PT cards was assessed for both whole blood and plasma tests. A calibration was carried out according to the World Health Organisation guidelines ${ }^{12}$ using blood samples from 20 normal subjects and 60 patients stabilised on oral anticoagulant therapy, each tested with TAS system (whole blood and plasma) and with BCT-441, the International Committee for Standardisation Haematology International Reference Preparation for human thromboplastin, which has an assigned ISI of $1.04 .^{13}$

\section{ANTI-Xa ASSAY}

Anti-Xa activity was determined using a clotting technique performed using an ACL instrument and commercially available reagents (Diagnostic Reagents Ltd) as previously described. ${ }^{14}$ The assay was calibrated using five dilutions of a pooled normal plasma to which was added the 4 th International Standard for unfractionated heparin at a final concentration of $0-1.0 \mathrm{iu} / \mathrm{ml}$.

\section{PRECISION OF INR DETERMINATIONS}

A normal sample was prepared by pooling citrated plasma from 20 normal subjects and an abnormal sample using plasma from five patients on warfarin. Each was stored at $-70^{\circ} \mathrm{C}$ after snap freezing on dry ice. These two samples were analysed on 10 different days to allow an assessment of between-assay precision. Within-assay precision was assessed by 10 immediate replicate tests on samples from normal subjects and patients on warfarin. In view of the time taken to complete 10 replicates by five techniques, different subjects were used for within-assay precision tests with each technique to limit in vitro sample changes, tests with one technique being completed before collection of samples for tests with another.

Between-assay and within-assay precision were assessed for TAS system and conventional thromboplastins, with the exception of between-assay precision for whole blood sam- ples on the TAS system. In each case the coefficient of variation (CV) was calculated (standard deviation $/$ mean $\times 100 \%)$ as an indication of precision.

\section{Results}

PRECISION

Between-assay (10 tests on different days) and within-assay (10 immediate replicate tests) precision results are shown in table 1 . Withinassay CVs ranged from $0.9 \%$ IL thromboplastin ACL instrument) to $9.7 \%$ (TAS system, whole blood), and between-assay CVs from $4.2 \%$ (IL/ACL) to $9.9 \%$ (MR, manual end point)

\section{NORMAL SUBJECTS}

Results of PTs of 20 normal subjects, used to calculate MNPT and derive INR for conventional thromboplastins, are shown in table 2 . There was no significant difference (paired $t$ test) between PTs for the TAS system when plasma or whole blood was used. The calculated MNPTs for TAS were 10.7 (plasma) and 10.6 (whole blood), which compares with manufacturer's values (encoded in test cards and used to derive INRs) of 10.9 and 11.4.

MEASURED OR UNMEASURED VOLUME OF BLOOD The mean INR of 10 samples analysed using a measured $35 \mu \mathrm{l}$ volume of blood was 1.97 compared with a mean INR of 2.00 for 10 samples applied as blood drops of unmeasured volume $(r=0.98$, paired $t$ test; $\mathrm{p}=0.59)$.

\section{PATIENTS ON WARFARIN}

Three plasma samples had INRs $>10.0$ with one or more techniques. These were excluded from statistical analyses. All TAS INR results described below were as determined with the manufacturer's ISI and MNPT unless otherwise stated.

Results of INRs determined with three conventional thromboplastins and with the TAS system are shown in table 3 . These were compared by the analysis of variance (ANOVA) method. The four groups of patients were considered separately and collectively. There were no significant differences between mean TAS results with whole blood or plasma either for individual patient groups or the whole group collectively. A Bland Altman ${ }^{15}$ plot of these data are shown in fig 1 .

The relations between INR results obtained with whole blood analysed using the TAS system and INRs of plasma determined using each of three conventional thromboplastins are shown in fig 2 (MR), fig 3 (IL), and fig 4 (DA).

\section{Initiation of warfarin treatment}

During initiation of warfarin treatment (without heparin) INR results obtained using the TAS system (using whole blood samples) were not significantly different (ANOVA) from those obtained with conventional thromboplastins.

\section{Combined warfarin and heparin}

INR results obtained during initiation with concurrent intravenous heparin infusion with the TAS system (whole blood) were less than 


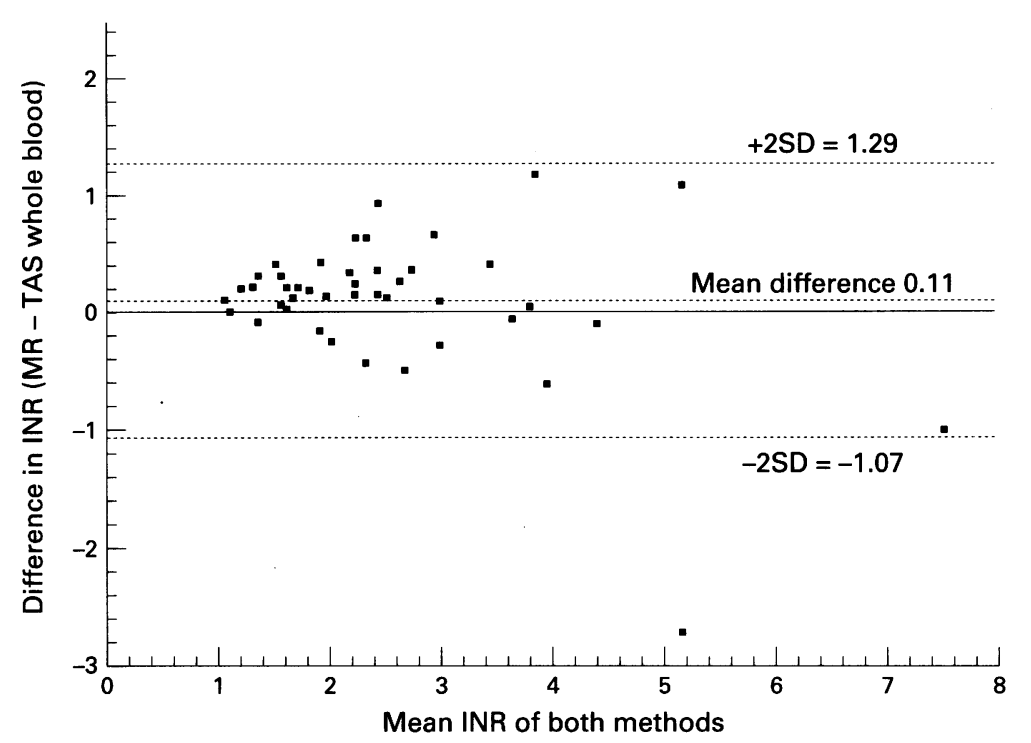

Figure 2 Difference in INRs determined with the TAS instrument (whole blood sample, manufacturer's ISI and MNPT) and with Manchester Reagent (plasma sample). The dotted lines are the mean (2 SD) of the differences in INR between the two methods. The solid line represents zero difference.

INR results obtained with MR (ANOVA; $\mathrm{p}<0.05$ ) or DA (ANOVA; $\mathrm{p}<0.01$ ) by $20 \%$ and $23 \%$, respectively. In this group the heparin concentration by anti-Xa assay was in the range $0.06-1.02 \mathrm{iu} / \mathrm{ml}$ with a mean result of $0.36 \mathrm{iu} / \mathrm{ml}$. There was no correlation between the anti-Xa activity and the difference between INRs for the five patients with INRs $<1.7$ (by TAS). In contrast, for the five patients with INRs > 1.7, the difference between INRs determined with DA and TAS (whole blood) increased as anti-Xa activity increased $(r=0.89, \mathrm{p}=0.04)$.

\section{Stabilised warfarin treatment}

During stabilised warfarin treatment, INRs obtained with the TAS system (using whole blood samples) were significantly less $(p<0.001)$ than results obtained with IL (by an average $22 \%$ ) or with DA (by an average $14 \%)$. There was no significant difference

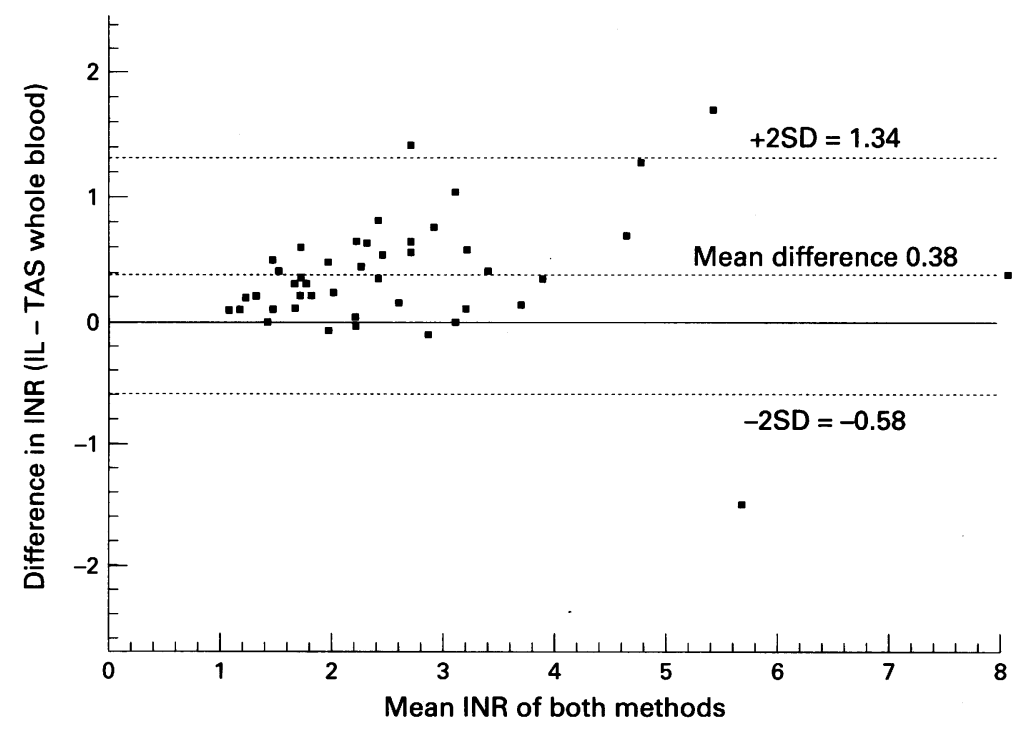

Figure 3 Difference in INRs determined with the TAS instrument (whole blood sample, manufacturer's ISI and MNPT) and with Instrumentation Laboratory PT Fib HS Plus reagent (plasma sample). The dotted lines are the mean (2 SD) of the differences in INR between the two methods. The solid line represents zero difference. between results obtained with the TAS instrument and MR.

RELATION BETWEEN INR OBTAINED WITH DIFFERENT CONVENTIONAL THROMBOPLASTINS Results obtained with MR were significantly $(\mathrm{p}<0.001$ ) lower (by an average $13 \%$ ) than those obtained with IL reagent in the stabilised patient group. For individual patient groups there were no other significant differences between results obtained with conventional thromboplastins.

\section{ALL PATIENT GROUPS COMBINED}

When data from all patient groups were combined $(n=45)$, excluding three patients with INRs of $>10.0$, results obtained with the TAS system (using whole blood) were significantly less than results obtained with IL (16\%, $\mathrm{p}<0.001)$ or DA $(11 \%, \mathrm{p}<0.05)$ reagents. Results obtained with MR were significantly less than those with IL $(11 \%, p<0.01)$. In contrast to data obtained with whole blood, results obtained with the TAS using plasma samples were not significantly different from those obtained with conventional thromboplastins when all patient groups were combined.

USE OF A LOCALLY DETERMINED MNPT

In addition to the TAS INR calculated automatically using manufacturer's MNPT and ISI, PTs obtained with the TAS instrument (whole blood) were converted into INR by substituting the locally determined MNPT (10.6) for the manufacturer's value (11.4) (table 3). When a local MNPT was employed there was an improvement in agreement between TAS (whole blood) and conventional reagents. The significant differences between TAS results and those obtained with DA (described above) within patient subgroups were abolished. Thus during stabilised warfarin the significant difference between INRs with TAS and DA was no longer present, and although the difference between TAS and IL remained significant (IL higher than TAS by $13 \%, \mathrm{p}<0.0001)$ agreement was improved. For all data combined $(n=45)$ there was no significant difference between TAS (whole blood) results and INRs obtained with conventional reagents.

DETERMINATION OF ISI OF TAS PT CARDS

Prothrombin times of normal subjects and patients on warfarin as determined with BCT441 were plotted against results obtained with the TAS system and plasma or whole blood samples according to WHO guidelines. ${ }^{12}$ There were no outlying points using the method of Tomenson and Thomson. ${ }^{16}$ The ISI of this batch of TAS PT cards based on data in our laboratory was 1.028 for plasma and 0.984 for whole blood samples (compared to manufacturer's values of 0.98 and 0.97 for plasma and whole blood, respectively). The coefficients of variation (calculated as SE/slope $\times 100 \%$ ) of the ISIs were $3.3 \%$ for both whole blood and plasma calculations. 


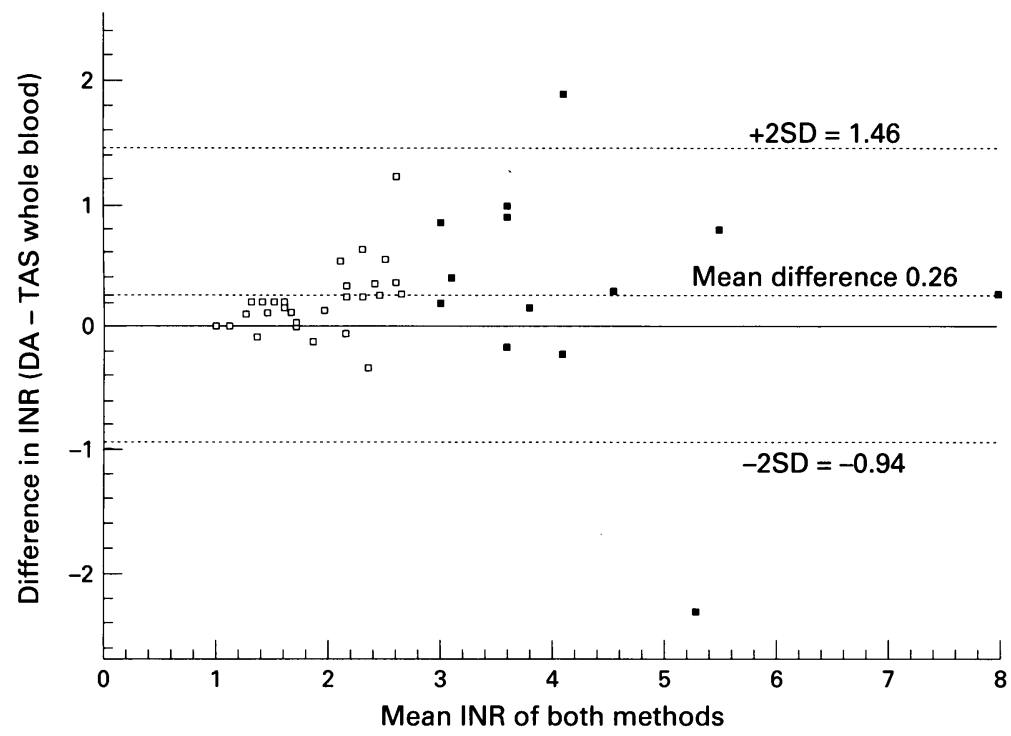

Figure 4 Difference in INRs determined with the TAS instrument (whole blood sample, manufacturer's ISI and MNPT) and with Diagen Activated thromboplastin (plasma sample). The dotted lines are the mean (2SD) of the differences in INR between the two methods. The solid line represents zero difference.

\section{Discussion}

Laboratory control of oral anticoagulant treatment without adequate reference to sensitivity of the laboratory technique and reagent may have an adverse effect on clinical management, ${ }^{16-19}$ and the INR system has undoubtedly contributed to improvement in this respect. ${ }^{4}$ Use of conventional thromboplastins for determination of INR within laboratories has been supplemented by the availability of portable near-patient testing instruments. ${ }^{5-8}{ }^{10}$ In the present study we evaluated one such analyser in respect of INR determinations of both citrated plasma and citrated venous whole blood and compared results to those obtained with conventional reagents and techniques.

Determination of INR using manual techniques is commonly associated with duplicate testing whereas the superior precision of some coagulometers has led to single testing in some cases. To evaluate precision of TAS INRs we determined within-assay and between-assay precision by replicate tests of identical samples. The within-assay CV for normal plasma and whole blood samples analysed using TAS were similar to those of conventional techniques for replicate determinations. The within assay $\mathrm{CV}$ of a warfarinised sample was higher for TAS (whole blood) than for conventional reagents. Between assay CV for plasma samples was similar for TAS and conventional reagents for both normal and warfarinised samples. The TAS results obtained here compare favourably with a between-assay $\mathrm{CV}$ of $18.8 \%$ for another near-patient test instrument noted by Tripodi and co-workers, ${ }^{8}$ and are similar to those of Rose and co-workers ${ }^{7}$ with the TAS instrument.

Whereas some other near-patient test systems $\mathrm{s}^{5}$ employ fingerstick capillary blood, the TAS is calibrated for use with citrated samples analysed either as intact whole blood or citrated platelet poor plasma following centrifugation. There was no significant difference between PTs of normal subjects or INRs of warfarinised patients when samples were analysed as whole blood and as plasma after centrifugation. Either sample type could therefore be used in routine practice.

The main principle of the INR system is that the same result should be obtained irrespective of the reagent used for INR determinations. Differences between INRs determined using different conventional thromboplastins have been reported. ${ }^{19} 20$

In the present study INRs determined on whole blood using the TAS instrument and manufacturer's MNPT were less than results obtained with DA and IL reagents. These differences were sufficient (11-16\%) to influence patient management in some cases and were similar in magnitude to differences present when two conventional reagents (MR and IL) included in the study were compared. These differences were greater at greater INRs. In contrast, results obtained on plasma samples (rather than whole blood) using the TAS analyser were similar to those obtained with conventional thromboplastins. Both ISI and MNPT values are encoded into test cards by the manufacturer. In the present study the TAS ISI values were assessed according to WHO recommended criteria ${ }^{12}$ and with the reference thromboplastin BCT-441. ${ }^{13}$ Calculated ISIs for plasma and whole blood were $4.8 \%$ and $1.4 \%$ greater than manufacturer's stated values, respectively. This essentially confirms the manufacturer's values, particularly for whole blood and therefore does not explain the difference in INR values between TAS whole blood and conventional thromboplastins. In the present study, the locally determined MNPT for whole blood samples was $10.6 \mathrm{sec}-$ onds compared to the manufacturer's value of 11.4 seconds. Use of the local MNPT increased INRs determined with the TAS and improved agreement with conventional reagents. The statistically significant differences between TAS results (whole blood; all patient groups combined) and either DA or IL were abolished. Within the subgroup of stabilised patients, TAS (whole blood) results did however remain significantly lower than results obtained with IL. This difference (13\%) was practically identical to that observed between MR and IL in this group. We have confirmed the manufacturer's ISI for TAS (whole blood) but the possibility that inaccurate assignment of ISI to conventional thromboplastins has contributed to the differences described cannot be excluded from our data.

We have demonstrated that the TAS system can be used for determination of INR using plasma or whole blood with the manufacturer's ISI. Agreement with INRs determined using conventional thromboplastins was improved by the use of a locally determined MNPT in our study, suggesting that this approach should be adopted where possible. Differences between INRs obtained with TAS and one commonly used UK conventional thromboplastin were present in a group of patients within the therapeutic range for INR but were similar to the difference between this and another conventional thromboplastin. 
The authors are grateful to Cardiovascular Diagnostics Inc (Research Triangle Park North Carolina, USA) and Diagnostic Testing Limited (Bury St Edmunds, UK) for provision of tes cards and the TAS instrument, and to Marilyn Thorpe for preparation of this manuscript.

1 Dalen JE, Hirsh J. American College of Chest Physician and National Heart Lung and Blood Institute national conference on antithrombotic therapy. Arch Intern Med 1986;146:462-72

2 Loeliger LA, van den Besselaar AMHP, Broekmans AW. Intensity of oral anticoagulation in patients monitored with various thromboplastins. N Engl f Med 1983;308:1228-9.

3 Denson KWE. Thromboplastin-sensitivity, precision and other characteristics. Clin Lab Haem 1988;10:315-28.

4 Loeliger EA, van den Besselaar AMHP, Lewis SM. Reliability and clinical impact of the normalisation of the prothrombin times on oral anticoagulant control. Thromb Haemost 1985;53:148-54.

5 Jennings I, Luddington RJ, Baglin T. Evaluation of the Ciba Corning Biotrak 512 coagulation monitor for the control of oral anticoagulant therapy. $\mathcal{F}$ Clin Pathol 1991;44:950-3.

6 Yano Y, Kambayasni J, Murata K, Shiba E, Saron M, Kawasaki $\mathrm{T}$, et al. Bedside monitoring of warfarin therapy by a whole blood capillary coagulation monitor. Thromb Research 1992;66:583-90.

7 Rose VI, Dermott SC, Murray BF, Melver MM, Higt KA Oberhardt BJ. Decentralised testing for prothrombin time and activated partial thromboplastin time using a dryand activated partial thromboplastin time using a drychemistry port

8 Tripodi A, Arbin AA, Chantarangkul V, Bettega D, Mannucci PM. Are capillary whole blood coagulation monitors suitable for the control or oral anticoagulan treatment by the International Normalised Ratio? Throm Haemostas 1993;70:921-4.

9 White RH, Becker DM, Gunther-Maher. Outpatient use of a portable International Ratio/prothrombin time monitor. South Med f 1994;87:206-10.

10 Foulis PR, Wallach PM, Adelman HM, Sanford BH McCain J, Reed D, et al. Performance of the Coumatrak system in a large anticoagulant clinic. Am $\mathcal{F}$ Clin Pathol 1995;103:98-102.

11 Oberhadt BJ, Dermott SC, Taylor M, Alkadi ZY, Abruzzin A, Gresalhi NJ. Dry reagent technology for rapid convenient measurements of blood coagulation and fibrinolysis. Clin Chem 1991;37:520-6.

12 WHO Expert Committee on Biological Standardisation. 33rd report. WHO Technical Report Series 1983;687: 81-105.

13 Thomson JM, Parby KV, Poller L. Calibration of BCT/441 The ICSH reference preparation for thromboplastin 1986;55 379-82.

14 Donnachie D, Kitchen S, Preston FE. Evaluation of a clotting anti-Xa assay for heparin. Br F Haematol 1994;86:33.

15 Bland JM, Altman DG. Statistical methods for assessing agreement between two methods of clinical assessment. Lancet 1986; i:307-10

16 Tomenson JA, Thomson JM. Standardisation of the prothrombin time. In: Thomson JM, ed. Blood coagulation and haemostasis. Edinburgh: Churchill Livingstone, 1985: and haem $370-409$.

17 Hirsh J, Levine M. Confusion over the therapeutic range for monitoring oral anticoagulant therapy in North America. Thromb Haemost 1988;59:129-32.

18 Bussey HI, Force FW, Bianco TM, Leonard AD. Reliance on prothrombin time ratio causes significant error in anticoagulation therapy. Arch Intern Med 1992; 152:278-82.

19 Ansell JD. Imprecision of prothrombin time monitoring of oral anticoagulation: a survey of hospital laboratories. $A m \mathcal{F}$ Clin Pathol 1992;98:237-9.

20 Kitchen S, Walker ID, Woods TAL, Preston FE. Thromboplastin related differences in the determination of International Normalised Ratio. Thromb Haemost 1994;72 426-9.

21 Kitchen S, Jennings I, Woods TAL, Walker ID, Preston FE. Two recombinant tissue factor reagents compared to conventional thromboplastins for determination of International Normalised Ratio: a 33 laboratory collaborative study. Thromb Haemost 1996;76:372-6. 